TUMOUR IMMUNOLOGY

\title{
Unmasking cancer cell character
}

Interferons are critical in mounting an effective antitumour response, and loss of interferon signalling can result in resistance to immunotherapies such as immune checkpoint blockade. Two new studies now show that cancer cells already contain sufficient quantities of immunostimulatory nucleic acids to trigger interferon signalling, increase antitumour immunity and overcome resistance to checkpoint blockade. Loss of function of the RNA-editing enzyme ADAR1 enables tumour cells to sense these innate ligands.

Adar1, which encodes an adenosine deaminase that binds to and limits the sensing of endogenous double-stranded RNA (dsRNA), had previously been identified by the team led by Nicholas Haining as a gene whose deletion could confer sensitivity to immunotherapy. In their latest study, Haining and colleagues showed that Adar1-null tumours grew normally in wildtype, immunocompetent animals but were profoundly sensitized to treatment with anti-PD1 antibodies in multiple transplantable tumour models. Adar1-null tumours showed increased infiltration of several types of immune cells, and Adar1-null melanoma cells showed upregulation of interferon-stimulated genes (ISGs) and cytokine and chemokine genes in response to interferons. Genetic ablation of genes encoding proteins that mediate interferon sensing, such as interferon- $\gamma$ receptor 1 (Ifgr 1 ), interferon- $\alpha / \beta$ receptor 2 (Ifnar2) and Stat1, eliminated the sensitivity of Adar1-null tumours to immunotherapy.

The authors thus hypothesized that, in cancer cells, in the absence of ADAR1, there might be an interferon-stimulated increase in RNA species (normally edited by this enzyme), which can in turn activate interferon signalling, induce an ISG transcriptional programme and trigger an immune response. Further experiments identified the dsRNA sensors MDA5 and

$\mathrm{PKR}$ as those responsible for

Adar1-null-associated enhanced sensitivity to immunotherapy and increased inflammation and immune infiltration.

Finally, the authors tested whether loss of ADAR1 was sufficient to overcome common mechanisms of resistance to checkpoint blockade. They generated mouse models of immunotherapy resistance by eliminating $B 2 m$, a component of the class I major histocompatibility complex (MHCI) that is involved in the presentation of peptide antigens to the immune system. Loss of ADAR1 in B2m-null tumours was associated with a significant increase in immune cell infiltration.

Similarly, in the second paper, McDonald and colleagues describe how tumour-derived interferons can result in chronic signalling that poises cells to respond to dsRNA accumulation and makes tumours susceptible to ADAR1 loss.

The authors analysed the expression profile of ISGs and found the presence of ISG signatures in tumours without infiltration of interferon-producing immune cells. This observation suggested that cancer cells per se can be a source of interferon production.

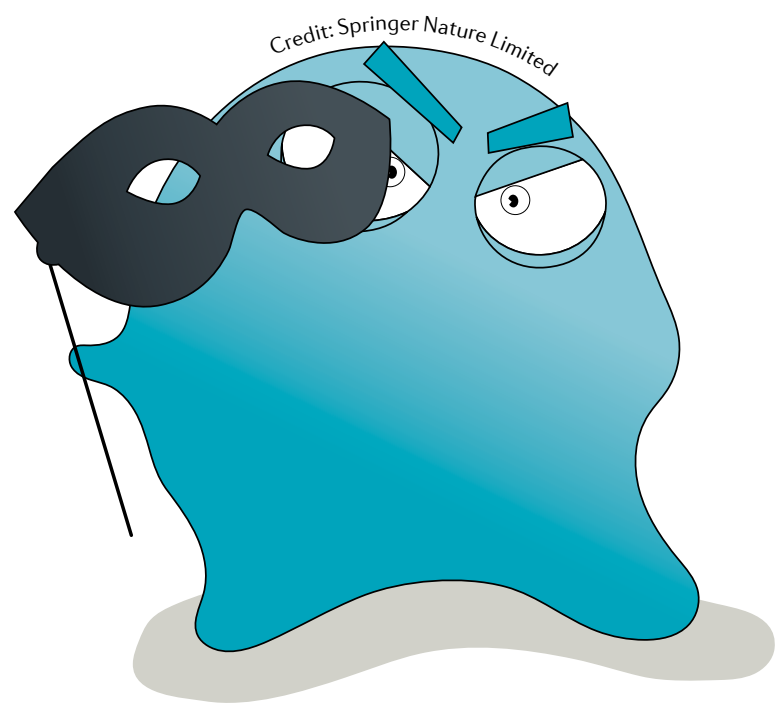

To confirm this hypothesis they established patient-derived tumour xenografts (PDXs) in nude mice that lack mature $\mathrm{T}$ cells, which enabled them to quantify interferons and ISGs and confirm whether these were from cancer cells (human transcripts) or from immune or stromal cells (mouse transcripts). The presence of the ISG signature correlated with the level of human type I and type III interferon transcripts, which confirmed that interferons produced by cancer cells can drive the ISG signature. Deletion and inhibition of key components of the interferon sensing and signalling pathway showed that cancer cell-intrinsic aberrant DNA species trigger STING-dependent interferon production that drives chronic ISG expression.

Understanding that this chronic ISG activation state may create new genetic vulnerabilities, the authors mined a large-scale pooled shRNA viability screen across 398 cancer cell lines and identified $A D A R 1$, an ISG, as the top hit whose depletion reduced the viability of ISG-expressing cancer cells. A further screen to identify the genes whose deletion would rescue the lethality observed following $A D A R 1$ deletion revealed that many of the components of the interferon pathway, such as MAVS, STING or IFNAR1, are required for the lethality induced by ADAR1 depletion.

These studies identify ADAR1 as an innate immune checkpoint and a novel target for combination with immune checkpoint blockade. They also suggest that other such checkpoints may exist, with similar effects on antitumour immunity.

\section{Teresa Villanueva, Senior Editor, Nature Reviews Drug Discovery \\ This article is modified from the original in Nat. Rev. Drug Discov.} (https://doi.org/10.1038/d41573-019-00005-y).

ORIGINAL ARTICLES Ishizuka, J. J. et al. Loss of ADAR1 in tumours overcomes resistance to immune checkpoint blockade. Nature 25, 95-102 (2019) | Liu, H. et al. Tumor-derived IFN triggers chronic pathway agonism and sensitivity to ADAR loss. Nat. Med. 25, 95-102 (2019) FURTHER READING Gannon, H. S. et al. Identification of ADAR1 adenosine deaminase dependency in a subset of cancer cells.

Nat. Commun. 9, 5450 (2018) 